\title{
La Investigación Científica en la Formación de Estudiantes Universitarios
}

\author{
Ligia Sánchez \\ ligia.uc@gmail.com \\ https://orcid.org/0000-0003-1410-9309 \\ Universidad de Carabobo. Campus La Morita \\ Maracay, Venezuela.
}

Maidelis Herrera

maidelis16@gmail.com

https://orcid.org/0000-0003-0634-3779

Universidad de Carabobo

Maracay, Venezuela.

Mairene Sánchez

mairenesanchez@gmail.com

https://orcid.org/0000-0001-9795-3075

Universidad Bicentenaria de Aragua

Maracay, Venezuela.

Recibido: 15/04/2020 Aceptado: 25/05/2020

\section{Resumen}

Se indagó respecto a la resistencia que tienen los estudiantes universitarios de involucrase en la actividad investigativa durante su proceso de formación universitaria. Se desarrolló una investigación de campo, desde una perspectiva cualitativa, soportada en la Fenomenología con estudiantes de la Facultad de Ciencias Económicas y Sociales, de la Universidad de Carabobo (Venezuela). Para la captura de la información se recurrió a los grupos de discusión y la observación participante. Se organizaron cinco grupos en total, tres con siete participantes y dos con seis; todos cursantes de las Escuelas de Administración Comercial y de Contaduría Pública. De los resultados emergió que la formación para la investigación científica, durante la carrera, se efectúa a través de las asignaturas del componente de investigación. Se captó que resistencia de los estudiantes a la actividad investigativa, durante su formación universitaria, está anclada a la existencia de una brecha, que se ha gestado entre la actividad investigativa que desarrollan los docentes en la institución y la actividad que los estudiantes realizan en sus TEG. Los estudiantes tienen poco conocimiento respecto a la actividad científica que se desarrolla en la universidad, lo cual limita su participación e involucramiento en dichas actividades y genera cierta aprehensión y temor producto de miedos e inseguridades, ya que la investigación se ve como algo reservado para los elegidos. Además, la poca motivación hacia la investigación, apareció relacionada a la experiencia vivida en las asignaturas del componente de investigación, la dinámica y la didáctica utilizada por la mayoría de los docentes; así como a la desarticulación entre estas asignaturas con las asignaturas del área disciplinar. A pesar de ello, los estudiantes consideraron que el ejecutar el proyecto de investigación, del Trabajo Especial de Grado, es relevante en su formación profesional, por su utilidad académica y su potencial para fortificar el conocimiento disciplinar.

Palabras clave: Investigación en la universidad, formación en investigación, Trabajo de grado 


\title{
Pesquisa Científica na Formação de Estudantes Universitários.
}

\begin{abstract}
Resumo
Foi pesquisado sobre a resistência dos estudantes universitários, no envolvimento das atividades de pesquisa, durante seu processo de formação universitária. Foi realizada uma pesquisa de campo, desde a abordagem qualitativa, com base na fenomenologia, com estudantes da Facultad de Ciencias Económicas y Sociales, da Universidad de Carabobo (Venezuela). A coleta de dados foi realizada com grupos de discussão e a observação participante. Foram organizados cinco grupos no total, sendo três com sete participantes e dois com seis. Todos estudantes das Escuelas de Administración Comercial y de Contaduría Pública. Os resultados apontam que a formação em pesquisa científica, durante a graduação, acontece por meio dos conteúdos das disciplinas de pesquisa. Os dados apontam que a resistência dos estudantes à atividade de pesquisa durante a sua formação universitária está associada à existência de uma lacuna que se criou entre a atividade de pesquisa, desenvolvida pelos docentes na instituição, e a atividade que os estudantes realizam em seus Trabajo Especial de Grado (TEG). Os estudantes possuem pouco conhecimento sobre a atividade científica que se desenvolve na universidade. Este fato limita sua participação e envolvimento nessas atividades e gera certa apreensão e temor, consequência de medos e inseguranças, já que a pesquisa é entendida como algo reservado aos escolhidos. Além disso, a pouca motivação para a pesquisa apareceu relacionada à experiência vivida nas disciplinas dos conteúdos de pesquisa, a dinâmica e a didática utilizada pela maioria dos professores, assim como a desarticulação entres estas disciplinas, com as disciplinas da área disciplinar. Apesar disto, os estudantes consideram que realizar o projeto de pesquisa do TEG é relevante na sua formação profissional, por sua utilidade acadêmica e seu potencial para fortalecer o conhecimento disciplinar.
\end{abstract}

Palavras-chaves: Pesquisa na Universidade; Formação em Pesquisa; Trabalho de Conclusão de Curso

\section{Scientific Research in the Training of University Students}

\begin{abstract}
I was investigated regarding the resistance that university students have to get involved in research activity during their university training process. A field research was developed, from a qualitative perspective, supported in Phenomenology with students from the Faculty of Economic and Social Sciences, from the University of Carabobo (Venezuela). Discussion groups and participant observation were used to capture the information. A total of five groups were organized, three with seven participants and two with six; all students from the Schools of Commercial Administration and Public Accounting. From the results it emerged that the training for scientific research, during the career, is carried out through the lectureships of the research component. It was noted that student resistance to research activity, during their university training, is anchored to the existence of a gap, which has arisen between the research activity carried out by professors in the institution and the activity that students carry out in their Special Degree Work. Students have little knowledge regarding the scientific activity that takes place in the university, which limits their participation and involvement in these activities and generates a certain apprehension and fear due to the unknown and
\end{abstract}


insecurities, since research is seen as something reserved for the "chosen ones". Furthermore, the low motivation towards research appeared related to the experience lived in the lectureships of the research component, the dynamics and didactics used by most of the professors; as well as the disarticulation between these lectureships with the ones of the disciplinary area. Despite this, the students considered that executing the final research project of the Special Degree Work is relevant in their professional training, due to its academic usefulness and its potential to strengthen disciplinary knowledge.

Keywords: Research at University, Research Training, Undergraduate work

\section{Introducción}

El acercamiento a la investigación científica de los estudiantes, durante el proceso de formación universitaria, constituye uno de los retos que se deben afrontaren la universidad, para lograr despertar el interés de los futuros profesionales por el quehacer investigativo y además impactar favorablemente la producción científica en las universidades. La incorporación de la investigación al proceso de formación universitaria de pregrado, nace en 1810 con la creación de la Universidad de Berlin, por iniciativa de Wilhelm von Humboldt (Barsky, 2017). Esto le agregó a la universidad un nuevo valor. A partir de su puesta en práctica se enriquecería la producción científica y a la vez que se robustecería la formación de los estudiantes universitarios, en virtud que la práctica científica va a favorecer su involucramiento con la generación de ciencia y les acercaría a la aplicación de procedimientos metodológicos científicos en la búsqueda de soluciones a problemas de su entorno.

Es decir, al ser la investigación uno de los ejes fundamentales del quehacer de la universidad, ésta se constituye en el espacio idóneo para la generación no solo de una cultura científica, sino además, de productos científicos vinculados con las necesidades del contexto social. En este sentido, el proceso de formación de profesionales universitarios necesariamente debería estar articulado a la acción investigativa, de modo que estas instituciones se constituyan en los principales entes potenciadores de la cultura científica, generadoras de la producción científica que la sociedad requiera en cada momento histórico. Es decir, se presupone que en la formación universitaria se forman los sujetos capaces de dar respuesta a los requerimientos que la sociedad demanda. (Flores \& Villegas, 2008).

En la realidad universitaria, casi en términos generales, el acercamiento de los estudiantes al quehacer científico, durante su formación disciplinar en las universidades, se logra fundamentalmente a través de la elaboración del Trabajo Especial de Grado (TEG). En Venezuela, el TEG constituye un requisito académico obligatorio, para lograr la obtención del 
grado de licenciatura en la mayoría de las instituciones de Educación Superior. Su realización abre la posibilidad de involucrar a los estudiantes en el desarrollo de un proyecto de investigación científica, donde se integra la revisión de avances teóricos con la aplicación de procedimientos metodológicos rigurosos, con el propósito de generar un producto intelectual concreto.

La preparación del estudiante para el ejercicio de su actividad investigativa se logra con el apoyo académico que se ofrece en las asignaturas del componente de investigación. En éstas el docente recurre a estrategias de aprendizaje para la enseñanza de cómo investigar las cuales, según Fernández (2015), son un factor de gran importancia al momento de favorecer el estímulo de los estudiantes para involucrarse en la ejecución del proyecto de investigación, orientado al TEG. Es importante destacar que la valoración de la producción de conocimientos, a través del TEG, queda expresado en la significación que los estudiantes le otorgan al proceso investigativo y al producto intelectual que de éste se genera, en su formación disciplinar (Herrera \& Sánchez).

Sin embargo, la realidad nos devela que la situación resulta compleja, tomando en cuenta que en ese proceso interactúan sinérgicamente diversos factores: los docentes, los estudiantes y las instituciones propiamente dichas. Una mirada hacia el factor docente, queda evidenciada en diversas investigaciones (Martínez, 1989; Castro-Silva 1997; Sabino, 2000 y Arias, 2008), en las cuales se señala que en las universidades venezolanas, los docentes universitarios no han considerado la investigación como un elemento fundamental, dentro de las actividades propias que deben desempeñar, además de la docencia, extensión y servicio; esto, por supuesto, incluye a los profesores de las asignaturas de metodología de la investigación. Sin embargo, resulta aun difícil la articulación de estos campos de acción con la investigación; en particular pues persiste en algunos docentes la creencia que es posible “...la docencia sin investigación, sin elementos de incorporación novedosos y, sobre todo, sin querer entender que la realidad nacional y mundial se mueve más rápido que nuestro campo de percepción..." (Flores \& Villegas 2008, p. 67). En este sentido, al no hacer investigación, se dificulta la efectividad del acto de enseñar a investigar. Por esta razón, se puede afirmar que éste es uno de los factores que limitan el involucramiento de los estudiantes con la actividad investigativa.

Por otro lado, no solamente se ha constatado la poca experiencia en investigación que 
tienen los docentes universitarios, sino además, éstos se aferran a esquemas rígidos al momento de hacer el seguimiento de los proyectos de investigación de sus estudiantes (Yapu, 2017). Cuando se recurre al “...principio de formar-investigando e investigar-formando se llega también a formar o fortalecer las competencias cognoscitivas o teóricas, técnicas y sociales e, incluso, éticas" (Yapu, ob.cit, p.34). Asimismo, no menos importante resulta el abordaje de esta problemática a partir de la consideración de las concepciones (Martínez, Harrington \& Bolívar, 2011) y representaciones (Sivira, 2014) que, sobre la investigación, arrastran los estudiantes. Éstas evidentemente también condicionan el acercamiento a la investigación científica de los estudiantes de educación superior. Es importante acotar que en el proceso formativo no solo se integra, apropia y transmite conocimientos (Páez, 2010), sino que además, este proceso se enriquece cuando se desarrolla de la mano de la actividad investigativa generadora de producción académica (Argüello, 2010).

El acercamiento de los estudiantes al quehacer científico, durante su formación universitaria, resulta en muchos casos una tarea traumática. Diferentes investigaciones lo han puesto de manifiesto (Abreu, 2015; Morales Rincón \&Tona, 2005) al ahondar en el síndrome de "todo menos tesis" (TMT). En este sentido, se ha develado que las causas del abandono de la formación universitaria han estado marcadas por la imposibilidad de culminar la realización del TEG. En este marco resalta la importancia de considerar la manera cómo se lleva a cabo la enseñanza del proceso de investigación (Sánchez, 2014) y los contenidos que se imparten en las asignaturas del componente investigativo. Al respecto hay quienes sostienen (Rodríguez, Méndez, González, Cantalapiedra \& González Polo, 2016), que se debe considerar no solo lo concerniente a la estructura o esquema del camino a recorrer para el diseño de un proyecto de investigación, sino además aspectos relativos a la comunicación de los resultados, es decir redacción, escritura de artículos científicos y también la presentación pública en eventos. Por otro lado, también es menester tomar en cuenta el impacto que tiene, durante el desarrollo del proyecto de investigación el acompañamiento al estudiante, bien sea bajo la forma de tutoría o asesoría, pues el rol del tutor es decisivo en el modo cómo se asume un proceso investigativo durante la formación universitaria (Gamero, 2014; Salinas, 1995). Es decir, dependiendo de esta experiencia se puede favorecer o no la valoración del acto investigativo. Este planteamiento se refuerza en lo expuesto por Castro-Gamero (2014), quien además agrega que el uso de la estrategia de tutoría en pequeños grupos, al momento de realizar el Trabajo de fin 
de carrera, deja muy buenos resultados en lo estudiantes, en la medida que se acude al trabajo cooperativo y la discusión grupal, lo cual le otorga un valor agregado al proceso, pues los estudiantes aprenden a trabajar, identificar problemas y buscar soluciones a los mismos cooperativamente.

Es importante resaltar el valor que cobra el rol del docente durante el proceso de involucramiento del estudiante con el quehacer científico, visto que su figura, en esta etapa de formación universitaria se perfila como articulador de la formación académica disciplinar con la formación científica, al lograr que el estudiante vincule los conocimientos adquiridos en las asignaturas del componente de investigación con la práctica científica en su disciplina. Asimismo, en este marco entra en juego, lo que Bolivar (2014), desde la Teoría de la Valoración reveló, al considerar de gran importancia los factores afectivos en la actitud de los estudiantes en el proceso de enseñanza-aprendizaje de la investigación. Este planteamiento, igualmente coloca el foco en el rol del docente, como factor determinante durante la formación investigativa de los estudiantes universitarios. De allí su consideración respecto a que en la enseñanza del proceso de investigación no solo es importante considerar "... la cognición, sino también los afectos, valoraciones, los juicios y apreciaciones que los aprendices puedan desarrollar..." (p.24)

Es evidente que la actitud de los estudiantes hacia las asignaturas del componente investigativo, a lo largo de su formación universitaria, constituye un elemento importante a considerar para lograr hurgar respecto a la resistencia que manifiestan éstos al momento de acercarse, como investigadores, al saber científico e identificar problemas de investigación, relacionados con la disciplina donde se desempeñarán, al culminar sus estudios universitarios.

De acuerdo a Yapu (2017), esta resistencia o rechazo a involucrarse con el quehacer investigativo responde, por una parte, a la forma cómo los docentes mantienen una actitud inflexible en el seguimiento de protocolos y esquemas que orientan su mirada o posición epistémica que asumen, los cuales se convierten en "camisas de fuerza", que actúan como líneas paradigmáticas o pautas perfectas e inalterables en el proceso de investigación. En este mismo orden de ideas, Chacón (2018) identificó dos aspectos importantes, por una parte que los docentes del componente de investigación no despiertan mayor interés hacia la investigación en los alumnos, pues las estrategias didácticas no involucran de manera práctica a los estudiantes en el quehacer investigativo. Asimismo, se ha podido develar la existencia de 
temores atávicos en los estudiantes, que bloquean la aproximación espontánea a la búsqueda de respuestas a problemas reales, en el campo de la disciplina donde ejercerán como profesionales, desde el saber científico (Herrera \& Sánchez, 2019). Es decir, los estudiantes no solo enfrentan problemas respecto a cómo se les enseña investigar, lo cual se transmite en las asignaturas del componente investigativo sino que, además, esto viene acompañado por los temores o mitos que socialmente se han construido sobre lo que representa investigar, todo lo cual genera aprehensión hacia todo lo que involucre la investigación científica.

Tomando en consideración los planteamientos anteriores se efectuó un cercamiento a la realidad de los estudiantes de los últimos semestres de la Facultad de Ciencias Económicas y Sociales de la Universidad de Carabobo (UC)-Campus La Morita, específicamente de la Escuela de Administración Comercial y Contaduría Pública, y se ha podido constatar desde la observación participante cierta resistencia o actitud, de los estudiantes, poco favorable a involucrarse en el proceso de investigación que obliga la realización del TEG. Sensibilizados ante esa realidad, que afecta tanto a los estudiantes como a la institución, se desarrolla la presente investigación con el propósito de hurgar respecto a los factores que están condicionando el interés de los estudiantes de participar en la actividad científica, a través de la realización del TEG. Es decir, develar elementos que sirven de asidero a la resistencia que tienen los estudiantes universitarios de involucrase en la actividad investigativa, durante su proceso de formación disciplinar, tomando en cuenta que, estando la universidad enmarcada en el aprendizaje por competencias, los estudiantes deberán desarrollar, de acuerdo a lo señalado por Castro-Gamero, (2014, p.271), “...la capacidad para aplicar la formación adquirida en contextos nuevos que requieren de una intervención creativa e innovadora...". Es decir, como bien agrega la autora, en la formación universitaria los estudiantes deberían lograr alcanzar las capacidades que les permita aportar respuestas a los requerimientos que la sociedad demande, desde el saber científico. De ahí la importancia que tiene el TEG, como parte del pensum académico, puesto que representa el cierre de la formación universitaria. Por lo tanto, ocupa un lugar fundamental dentro del proceso formativo.

\section{Aproximación a la realidad}

Para el acercamiento a la realidad se recurrió a la observación participante y a los grupos de discusión (Callejo, 2001; Valles, 2000). En tal sentido, se logró conformar cinco grupos, tres con siete participantes y dos con seis. Todos fueron estructurados a partir de la 
incorporación voluntaria de estudiantes cursantes del noveno y décimo semestre de Administración Comercial y Contaduría Pública del Campus La Morita. Como criterio de inclusión se mantuvo que los estudiantes hubiesen cursado las asignaturas del componente investigativo (Metodología de Investigación I, Metodología de Investigación II e Investigación Aplicada). La recogida de la información tuvo lugar en los espacios del Campus, durante tres semestres académicos de los años lectivos 2016, 2017 y 2018. Todas las sesiones de discusión fueron grabadas, respetando la privacidad, confidencialidad y previo consentimiento informado de los participantes, con lo que se garantizó el cumplimiento de los criterios éticos de protección de los estudiantes que participaron. (Valles, 2000).

Es importante acotar que la problemática emerge del propio sentir de los docentes de las asignaturas del componente de investigación, quienes han podido percibir poco interés en los estudiantes respecto a la realización del TEG, a pesar que éste es un condicionante para alcanzar el grado académico de licenciatura. En este sentido, surge la necesidad de develar las razones de la resistencia de los estudiantes a involucrarse en la actividad científica, durante su proceso formación disciplinar, a través de la ejecución del TEG, lo cual no solo impacta negativamente a los estudiantes sino también a la institución, en virtud que los TEG constituyen parte del producto intelectual que se genera desde las universidades.

La información recogida en los grupos de discusión fue procesada manualmente, codificando, categorizando y comparando de forma continua la información recogida en el discurso de los estudiantes.

\section{Resultados}

A efectos de garantizar el anonimato de los participantes, los fragmentos de los discursos se identificaron con nombres ficticios.

Al abordar en los grupos de discusión el tema de la investigación en su formación universitaria, emergió del discurso cinco dimensiones resaltantes y de gran interés para los estudiantes. Estas dimensiones fueron: 1) percepción que tienen los estudiantes sobre la investigación en la universidad, 2) valoración del involucramiento en un proyecto de investigación, 3) significado del TEG, para su formación disciplinar, 4) pertinencia de los contenidos de las asignaturas del componente de investigación, 5) la orientación académica para el diseño del proyecto de investigación y 6) divulgación de la actividad científica y sus productos. A continuación se recogen los discursos referidos a los aspectos antes 
mencionados:

\section{Percepción que tienen los estudiantes sobre la investigación en la universidad}

Al hurgar en el sentir de los estudiantes respecto a cómo ellos perciben la actividad investigativa en el Campus, en general se captó desconocimiento sobre lo que se investiga en la universidad. Esto llama la atención, pues en el campus se cuenta con figuras docentes acreditadas como investigadores con la más alta categoría en el Programa Nacional de Promoción y Estímulo a la Investigación y la Innovación (PEII) del Ministerio del Poder Popular de Ciencia, Tecnología e Innovación. En el Cuadro 1, se hace referencia a expresiones que destacan lo antes señalado.

Cuadro 1. Percepción de los estudiantes sobre la investigación en la universidad

\begin{tabular}{|c|c|}
\hline Dimensión & Expresión discursiva de los estudiantes \\
\hline \multirow{5}{*}{$\begin{array}{l}\text { Investigación en } \\
\text { la universidad }\end{array}$} & $\begin{array}{l}\text { "...A veces dicen algunos compañeros...esa profesora o profesor dicen que es } \\
\text { investigador o investigadora. Y yo les pregunto, ¿pero qué hace? Y me } \\
\text { responden yo no sé, escribe artículos en revistas científicas. (Diamante) }\end{array}$ \\
\hline & $\begin{array}{l}\text { “...Es verdad... a mi el otro día me mostraron a uno (Profesor)... que no } \\
\text { conozco,... porque él no me dio clase... y me dijeron que escribió un libro. Pero } \\
\text { yo me quedé en el sitio,... What??, Y le dije: ¿por qué ni siquiera ponen eso en } \\
\text { venta aquí?,... o dicen algo... Hay no sé... es que esas cosas son como de ellos. } \\
\text { A uno no le dicen en clase nada de eso. Como por ejemplo: miren muchachos, } \\
\text { busquen este libro que es del profe tal, o...léete este artículo que es del profe X } \\
\text {...Sí, tenemos buenos profesores, investigan, pero no nos involucran en nada de } \\
\text { eso...queda como en un círculo cerrado y reducido... "(Perla) }\end{array}$ \\
\hline & $\begin{array}{l}\text { “... Yo creo que aquí, ni los docentes le dan mucha importancia a eso de } \\
\text { investigar y escribir libros, artículos científicos o tener un grupo de } \\
\text { investigación, porque... es que nunca se habla de eso... Bueno al menos yo no } \\
\text { tengo información sobre eso...(Rubí). }\end{array}$ \\
\hline & $\begin{array}{l}\text { "Disculpa... Bueno... Yo sé que se hace una, que otra cosa de investigación... } \\
\text { En una cartelera del hexágono siempre colocan un cronograma de charlas, ...o } \\
\text { algo así. Es un seminario. Mi profe de Métodos nos ha invitado. Alli discuten } \\
\text { sobre investigaciones que hacen... Yo asistí a una charla y me gustó. Pero habia } \\
\text { más gente de otras universidades que del Campus. Eso me llamó la atención... } \\
\text { también es que no nos enteramos. Fíjate quiénes de los que estamos aquí han } \\
\text { visto esa cartelera?. (Oro) }\end{array}$ \\
\hline & $\begin{array}{l}\text { “Verdad, yo no leo carteleras... Es que eso de investigar creo que a mí no se... } \\
\text { es como mucho. Para uno en la universidad no creo que deje mucho. Pienso en } \\
\text { eso y me da flojera, ...Ayyy no!! ... porque es que de eso se habla solamente } \\
\text { cuando comenzamos en primer semestre y después más nunca se habla de eso, } \\
\text { sino en el octavo semestre, cuando cursamos Metodología de la Investigación } \\
\text { II..." (Zafiro) }\end{array}$ \\
\hline
\end{tabular}

Continúa ... 
Cuadro 1. Percepción de los estudiantes sobre la investigación en la universidad (Continuación)

\begin{tabular}{|c|c|}
\hline Dimensión & Expresión discursiva de los estudiantes \\
\hline \multirow{5}{*}{$\begin{array}{l}\text { Investigación en } \\
\text { la universidad }\end{array}$} & $\begin{array}{l}\text { "Bueno... aquí de eso no se habla mucho es verdad. Tú me preguntas en qué } \\
\text { línea de investigación vas a hacer tu proyecto de TEG y...mira yo te juro que no } \\
\text { sé qué responder... Dime quién de ustedes lo sabe?...(Risas) El que lo sepa es el } \\
\text { genio de la lámpara....(risas)”"(Amatista) }\end{array}$ \\
\hline & $\begin{array}{l}\text { "En relación a las líneas de investigación... creo que, hay líneas de } \\
\text { investigación, eso lo dijo una vez el profe de Métodos,... creo que son como } \\
\text { quince,... no estoy seguro. Me mostraron el otro día una lista. Yo no soy el } \\
\text { genio... Pero más o menos séen lo que lo hare... (TEG) en algo de recursos } \\
\text { humanos...eso me gusta. Y sobre eso hay una línea, yo lo vi en la lista. El } \\
\text { problema es buscar el profesor que trabaje en esa línea. Eso... sí... es verdad } \\
\text { que se desconoce Mmmm...falta que nos informen más sobre eso también..." } \\
(\text { Oro) }\end{array}$ \\
\hline & $\begin{array}{l}\text { "Me das la razón... genio de la lámpara!!! (risas)... Qué tanto sabes.!! Por lo } \\
\text { que dices es Muy poco. Esa respuesta me dice que sabes tanto como yo,... y ya } \\
\text { estamos terminando octavo semestre!!. Eso es preocupante. Bueno a mí me } \\
\text { preocupa, no se a ti. No sé aún qué haré, ni quien será mi tutor!!!... Es que el } \\
\text { problema es no saber nada sobre lo que investigan los profes... Ese es el } \\
\text { problema... Y ellos (los docentes) son los que saben de } \\
\text { eso(investigar)"(Amatista) }\end{array}$ \\
\hline & $\begin{array}{l}\text { "Mira, mira...perdón...sí se está investigando en el Campus..., pero el } \\
\text { problemas es, que no sabemos quiénes investigan, cuáles son los grupos que } \\
\text { existen, dónde están? ... Eso es muy importante que lo sepamos...porque eso } \\
\text { nos ayudaría mucho cuando vamos a identificar el problema de investigación y } \\
\text { luego a desarrollar el proyecto. Eso de estar solos en el proyecto no nos gusta. } \\
\text { Fíjate que Nosotros siempre buscamos hacerlo de tres. Esoes buscando } \\
\text { apoyo...No sé i!..., aunque sea en compañeros..."(Jade). }\end{array}$ \\
\hline & $\begin{array}{l}\text { "A decir verdad, investigar queda como algo entre ellos (los docentes)... A } \\
\text { veces pienso que no quieren que entremos en su mundo...eso no me gusta...Y me } \\
\text { pregunto: ¿Por qué entonces quieren que uno investigue y haga el TEG... } \\
\text { Entonces muéstrame cómo tú lo haces...verdad???"(Jaspe) }\end{array}$ \\
\hline
\end{tabular}

Fuente: Grupo de Discusión

Las expresiones antes expuestas revelan que la percepción que tienen los estudiantes respecto a la investigación en la universidad, está relacionada sólo a la práctica de ésta por parte de los docentes. Podríamos deducir que los estudiantes ven en la figura de sus docentes a los entes llamados a generar ciencia y, por tanto, echan en falta que no se les informe sobre lo que hacen sus profesores al respecto. Es una realidad que no son muchos los docentes universitarios que se dedican a la investigación. Esta falencia queda evidenciada en el discurso. Es decir, por una parte, son pocos los docentes que investigan y, por la otra, la 
información sobre lo que hacen es limitada, lo que pudiera estar evidenciando debilidad institucional en la divulgación de la actividad investigativa en la universidad. Esta situación pueda afectar el interés de los estudiantes hacia el acto investigativo. De ese modo, se puede afectar el interés de los estudiantes hacia la acción de investigar y ser sujeto protagonista de este proceso. En efecto, aún no se le otorga, en la universidad, al acto de investigar el lugar privilegiado que deben tener como actividad propia de todos los docentes y estudiantes (Sabino, 2000 y Arias, 2008). Cabe señalar, que se está dejando de lado el principio que reza que solo investigando se forma y solo formando se investiga, tal como sostiene Yapu (2017).

La investigación en la universidad, entonces es percibida por los estudiantes como una actividad que, en el campus, no tiene mucha relevancia o pertenece al mundo de los escogidos para tal fin. Se percibe que sólo pocos entran al mundo de la ciencia en la universidad.

\section{Valoración del involucramiento de los estudiantes en la actividad investigativa.}

Casi todos los estudiantes, echan en falta que no participan en actividades científicas o proyectos de investigación que se desarrollan en la universidad. Y tampoco participan en eventos científicos, que tuvieran lugar en otras facultades u otros centros de educación universitaria de la región; sus discursos pueden ser apreciados en el Cuadro 2.

Cuadro 2. Valoración de los estudiantes de su involucramiento en la actividad investigativa

\begin{tabular}{|l|l|}
\hline Dimensión & Expresión discursiva de los estudiantes \\
\hline & $\begin{array}{l}\text { "Para mí, cuando sali de bachillerato, donde hice mi proyecto de quinto } \\
\text { año, soñaba con eso de investigar. Y mi primer semestre en la UC me } \\
\text { motivó mucho hacia eso, gracias a mi profa de Métodos I. Ella hablaba } \\
\text { con pasión de entrar al mundo de la ciencia, ...cómo ella se inició en eso } \\
\text { de investigar. Y le dije que yo quería hacer eso: investigar. Recuerdo que } \\
\text { nos dijo que todo lo que se aprendía en esa materia teníamos que } \\
\text { aplicarlo en todas las materias... y eso es verdad, aunque no es fácil } \\
\text { Invlicarlo (risas)... "(Oro). }\end{array}$ \\
\cline { 2 - 3 } $\begin{array}{l}\text { Ios estudiantes en la } \\
\text { actividad } \\
\text { investigativa. }\end{array}$ & $\begin{array}{l}\text { “...Una vez la profe de Métodos I dijo en el salón de clases que quería } \\
\text { estudiantes para incorporarlos a un proyecto de investigación...le } \\
\text { pregunté si pagaban y me dijo que no...que el aprendizaje sería la } \\
\text { retribución. Yo fui a la Unidad de Investigación. Así fue... he aprendido } \\
\text { mucho, porque en las reuniones discuten sobre el instrumento de } \\
\text { recolección de datos, la recolección de datos". (Berilio) }\end{array}$ \\
\hline
\end{tabular}


Cuadro 2. Valoración de los estudiantes de su involucramiento en la actividad investigativa

\begin{tabular}{|c|c|}
\hline Dimensión & Expresión discursiva de los estudiantes \\
\hline \multirow{6}{*}{$\begin{array}{l}\text { Involucramiento de } \\
\text { los estudiantes en la } \\
\text { actividad } \\
\text { investigativa. }\end{array}$} & $\begin{array}{l}\text { “... Si... yo cursé contigo....imagínate hace como siete semestres...Dijo } \\
\text { también que buscaban un beca-servicio en la Unidad de } \\
\text { Investigación....Yo siempre me acercaba por allá y desde el tercer } \\
\text { semestretrabajo como beca servicio allí. Y apoyo en transcribir y } \\
\text { ordenar datos...ayudo a los profes a buscar información sobre sus } \\
\text { proyectos,... a actualizar sus curriculos para el PEI... he aprendido } \\
\text { mucho..”.(Aurita) }\end{array}$ \\
\hline & $\begin{array}{l}\text { "Para mí... al cursar Métodos II, me gustó mucho cómo daba la clase la } \\
\text { profa.Estoy haciendo mi proyecto de TEG con ella y estoy feliz... Me dijo } \\
\text { estoy investigando sobre este tema....y yo le dije...yo quiero trabajar con } \\
\text { Ud... O sea, es su proyecto, pero yo estoy sacando el mio } \\
\text { también..."(Topacio). }\end{array}$ \\
\hline & $\begin{array}{l}\text { "Algunos estudiantes pueden tener suerte y caerle bien a un profe... y } \\
\text { éste lo incorpora en su proyecto o en alguna cosa de investigación. Pero } \\
\text { eso no es lo común... Ojo!!!... Yo hubiera querido tener el } \\
\text { privilegio, Ufff... pero a veces eso es solo con los preparadores } \\
\text { (estudiante que apoya al docente) que tienen esa fortuna..." Yo llegué a } \\
\text { oír, cuando cursaba segundo semestre, que al menos en matemáticas } \\
\text { unos andaban en talleres con manejo de calculadoras y eso...de un } \\
\text { proyecto de investigación de un profe....pero puros preparadores de } \\
\text { Mate...!! (Zafiro) }\end{array}$ \\
\hline & $\begin{array}{l}\text { “...Eso de formar parte del grupo que investiga con el profe suena } \\
\text { bonito. Te imaginas si fuera lo establecido?, lo obligatorio.?.... No } \\
\text { tendriamos problemas en el noveno semestre para...bueno...hacer el } \\
\text { proyecto nuestro de TEG...este estrés que nos mata...(Risas). No } \\
\text { buscaríamos "Ayuda" por fuera con gente que nos saca los ojos } \\
\text { cobrando "asesoria"....Hayyy mija sería otra realidad..."(Lapizlasuli). }\end{array}$ \\
\hline & $\begin{array}{l}\text { "Yo,... por lo menos, no me he enterado de ningún caso de profesores } \\
\text { que busquen estudiantes para participar en un proyecto de } \\
\text { investigación..."(Esmeralda). }\end{array}$ \\
\hline & $\begin{array}{l}\text { “Me gustaría conocer la lista de proyectos de investigación que se están } \\
\text { desarrollando, a ver si puedo yo participar alli y hacer mi TEG con } \\
\text { ellos... Me parece mezquino que no se conozca nada de eso. Los } \\
\text { estudiantes necesitamos saber a qué profesores podemos acudir para } \\
\text { investigar... Bueno esteee...eso parece un sueño...(Risas)”.(Granate) }\end{array}$ \\
\hline
\end{tabular}

Continúa ... 
Cuadro 2. Valoración de los estudiantes de su involucramiento en la actividad investigativa

\begin{tabular}{|c|c|}
\hline \multirow{3}{*}{$\begin{array}{l}\text { Involucramiento de } \\
\text { los estudiantes en la } \\
\text { actividad } \\
\text { investigativa. }\end{array}$} & $\begin{array}{l}\text { "Bueno... tampoco hay que echarle toda la culpa al docente. La } \\
\text { institución debe tener una política clara al respecto. Si exiges TEG a los } \\
\text { estudiantes, tienen que darle también éste... no sé... la forma para que } \\
\text { podamos hacerlo sin trauma... porque esto es de verdad estresante..." } \\
\text { (Zafiro). }\end{array}$ \\
\hline & $\begin{array}{l}\text { "Realmente... yo no persigo formar parte de un grupo de investigación, } \\
\text { pero no estaría mal saber eso que hacen esos grupos de cientificos.... } \\
\text { Porque en general uno piensa más en la gente de Ciencias de la Salud. } \\
\text { Ellos si tienen laboratorios (risas)...". (Pirita) }\end{array}$ \\
\hline & $\begin{array}{l}\text { “...Para mi ésa sería una gran oportunidad porque ya tendría un } \\
\text { tema...o sea... proyecto, esteee... y así no tendría este estrés y el miedo } \\
\text { en escoger cuál tema ... será para mi TEG...que aún no lo tengo... por } \\
\text { cierto y estoy terminado el octavo semestre”(Espinela) }\end{array}$ \\
\hline
\end{tabular}

Fuente: Grupo de Discusión

Tal como lo expresan los estudiantes, existe una distancia entre lo que realizan los docentes como investigadores y la posibilidad de que los estudiantes se inserten en ese importante proceso. La incorporación de los estudiantes, en los proyectos de investigación que desarrollan los docentes, sería un factor altamente favorecedor en la formación universitaria y, además, pudiera contribuir el acercamiento de los estudiantes a la producción científica de la disciplina donde se están formando.

Si los estudiantes se articularan a la actividad científica durante el proceso de formación universitaria, la realización del TEG, podría representar una experiencia altamente gratificadora, resultado de su ingenio, lo cual le otorgaría un gran valor adicional. Con ello se rompería con las concepciones y representaciones (Martínez, Harrington \& Bolívar, 2011; Sivira, 2014 ) negativas que se han gestado en el imaginario y que arrastran los estudiantes universitarios respecto a la actividad investigativa. Romper con estas concepciones es una tarea pendiente a la cual debemos abocarnos para superar los temores que identificaron en los estudiantes Herrera \& Sánchez (2019), para asumir el reto y la responsabilidad de realizar su TEG.

\section{Significado del TEG, para su formación disciplinar}

En Venezuela es casi generalizada la obligatoriedad, en los estudios de educación superior, de realizar el TEG para optar al grado académico de licenciatura. Ahora bien, siempre resulta 
interesante sumergirse en lo que los estudiantes universitarios dicen al respecto. En el Cuadro 3 se recogen algunas impresiones respecto a lo que el TEG representa en su formación disciplinar de los estudiantes universitarios que participaron en el estudio:

Cuadro 3. Significado del TEG para su formación disciplinar

\begin{tabular}{|c|c|}
\hline Dimensión & Expresión discursiva de los estudiantes \\
\hline \multirow{7}{*}{$\begin{array}{l}\text { TEG y formación } \\
\text { disciplinar }\end{array}$} & $\begin{array}{l}\text { "...Más que aportarme algo para mi formación como contador... creo } \\
\text { que es algo más bien impuesto. Y así,... como lo hacemos ahorita } \\
\text { no..no...no sé...No considero que me ayude mucho..." (Lapizlasuli). }\end{array}$ \\
\hline & $\begin{array}{l}\text { "Yo creo que si ayuda a la formación como Contador Público... porque } \\
\text { buscamos profundizar sobre algo en particular, ...claro con alguien que } \\
\text { nos guíe a ampliar el contenido a indagar nos facilita una base teórica } \\
\text { relacionada muchas veces con las pautas para la realización del trabajo } \\
\text { de grado".(Rutilo) }\end{array}$ \\
\hline & $\begin{array}{l}\text { "Claro que cada asignatura da un contenido específico, ...bueno... eso } \\
\text { debería estar como relacionado... me imagino algo asi como si fuera } \\
\text { más profundo cuando hacemos el TEG ..."Zircon). }\end{array}$ \\
\hline & $\begin{array}{l}\text { "...Yo si considero que necesitamos en nuestra formación como } \\
\text { administradores o contadores, que sepamos cómo investigar sobre algo. } \\
\text { Por ejemplo si tú haces pasantía eso te puede ayudar como práctica, pero } \\
\text { si no la haces la experiencia de hacer el proyecto te puede ayudar a } \\
\text { saber más sobre algún problema en específico... al menos... Digo yo...!" } \\
\text { (Cinabrio) }\end{array}$ \\
\hline & $\begin{array}{l}\text { "Bueno... yo trabajo y quiero hacer mi Proyecto sobre algún problema de } \\
\text { la empresa. Mi jefe me dijo eso, ...quiere que me ocupe de algo que les } \\
\text { interese...Entonces ves que si te ayuda (el TEG) en tu práctica } \\
\text { profesional..."(Euclasa) }\end{array}$ \\
\hline & $\begin{array}{l}\text { "Pienso que a través del TEG podemos conocer... y ver cosas de } \\
\text { algunas empresas,...Bueno... o sea...situaciones donde podemos apoyar } \\
\text { con algo, no se...recomendar..." (Topacio) }\end{array}$ \\
\hline & $\begin{array}{l}\text { "Yo...termino ese peso... salgo de eso.. Eso es puro gasto de tiempo la } \\
\text { tesis y listo... me quita tiempo y dinero. Prefiero que me pongan en una } \\
\text { pasantía en empresa..." (Flourita) }\end{array}$ \\
\hline
\end{tabular}

Fuente: Grupo de discusión.

Si bien es cierto que Espig y Silva (2013) y González (2014), reportaron que era muy importante la investigación durante la formación de los estudiantes de carrereas de las ciencias de la salud, eso aplica también para los estudiantes de ciencias sociales, en vista que también 
la práctica investigativa les aporta mayor solidez en su formación disciplinar, porque les involucra con escenarios de la vida real, a los que tendrán que dar respuesta desde una mirada científica y rigurosa. El aprendizaje disciplinar investigando le podría aportar, a los futuros profesionales de Administración Comercial y Contaduría Pública, el desarrollo de sus habilidades analíticas, el cuestionamiento de ciertos procedimientos y la posibilidad de reflexionar críticamente sobre la búsqueda de salidas viables a situaciones complejas, dentro del entorno laboral donde se desempeñen. El desarrollo del espíritu científico necesita de ese ejercicio permanente y colectivo, que se puede robustecer durante el proceso de formación universitaria de los estudiantes de las ciencias sociales. Eso ya está demostrado en las ciencias puras. Así se procede generalmente en la formación de biólogos, médicos, físicos, químicos e incluso en la formación de médicos.

A pesar que en la facultad de Ciencias Económicas y Sociales no es una práctica común la realización de la pasantía, la emergencia en el discurso, de la importancia de la realización de pasantías resulta interesante, porque permite deducir que los estudiantes lo asocian con el ejercicio de su profesión, con el acercamiento práctico a su disciplina, con la posibilidad de un aprendizaje en la práctica, cosa que con el TEG se debería intentar lograr y hacer ver o sentir en los estudiantes. Es importante procurar que el TEG sea asumido con la responsabilidad y el compromiso que caracteriza la realización o ejecución de un trabajo científico, de este modo el aprendizaje será doblemente valioso. Sin embargo aun el TEG no es considerado, por la generalidad de los estudiantes, como la oportunidad de abordaje de un problema real concreto, identificado en el contexto, como algo que amerita ser investigado para lograr ser superado o para generar posibles respuestas de acción para su solución. Es decir como el espacio donde a través de la investigación se articula el conocimiento teórico con la práctica.

\section{Pertinencia de los contenidos de las asignaturas del componente de investigación.}

Los contenidos de los programas de las asignaturas del componente investigación en la formación universitaria, en términos generales, son casi idénticos para todas las carreras. Si se realiza una revisión sistemática de esos contenidos, se podrá constatar dicha afirmación. De igual manera al revisar la bibliografía sugerida en los mismos, se podrá evidenciar que se manejan casi las mismas referencias. Esto revela que hay cierta homogeneidad en el ámbito universitario, en cuanto al contenido que se imparte en estas asignaturas, para formar a los 
estudiantes universitarios en el ámbito investigativo.

Es decir, en cuanto a los contenidos programáticos no hay mayores observaciones. La estructura curricular lleva una secuencia lógica, que va desde la etapa básica inicial, de lo que es conocimiento, ciencia, el proceso de conocimiento científico, tipos de investigación científica y su diferenciación del conocimiento vulgar, la investigación documental y la búsqueda de información para el arqueo bibliográfico, tipos de fuentes y normas para citar y referenciar cada una de ellas. Para luego entrar en lo que es propiamente el diseño de la investigación y sus etapas, profundizando en cada uno de los elementos que corresponde abordar en cada una de ellas. Todo esto, con el firme propósito que los estudiantes adquieran las herramientas necesarias para que puedan asumir el diseño y desarrollo de un proyecto de investigación orientado a la realización del TEG. En el discurso de los estudiantes emergió fundamentalmente asuntos referidos a la forma cómo se imparte el conocimiento de los contenidos, es decir cómo se enseña a investigar. En el Cuadro 4 se incluyen fragmentos de los discursos de los estudiantes.

Cuadro 4. Pertinencia de los contenidos de las asignaturas del componente de investigación.

\begin{tabular}{|c|c|}
\hline Dimensión & Expresión discursiva de los estudiantes \\
\hline \multirow{5}{*}{$\begin{array}{l}\text { Pertinencia de los } \\
\text { contenidos de las } \\
\text { asignaturas del } \\
\text { componente de } \\
\text { investigación, }\end{array}$} & $\begin{array}{l}\text { "En Métodos II es donde nos dicen todo lo del proyecto de investigación, } \\
\text {...porque en Investigación Aplicada nada... puro revisar lo que } \\
\text { entregamos ..." Es contradictorio.(Jade) }\end{array}$ \\
\hline & $\begin{array}{l}\text { "Métodos I es todo como buscar en las tesis, libros y revistas, etc". } \\
\text { (Granate) }\end{array}$ \\
\hline & $\begin{array}{l}\text { “... Caramba!! ... que memoria!! Yo ni recuerdo que vi en Método I.. } \\
\text { Solo sé que. Nada, nada. No creo que aprendí nada... Disculpa... pero es } \\
\text { así... el prof. Puro bla, bla,bla,bla... nunca aterrizó. Cómo la pasé?... no } \\
\text { sé... no me pregunten...”(risas).(Pirita) }\end{array}$ \\
\hline & $\begin{array}{l}\text { “...Para mi Métodos II es el más importante... allí no me aburrí } \\
\text { mucho”(Aurita) }\end{array}$ \\
\hline & $\begin{array}{l}\text { “...Uno va con mucha ilusión a Métodos II porque cree que alli armará } \\
\text { el proyecto del TEG, ...pero eso es pura teoría...(risas)El problema lo } \\
\text { tienes después...estee ¿cómo pones en práctica todo ese poco de } \\
\text { cosas...?”(Cuarzo) }\end{array}$ \\
\hline
\end{tabular}

Continúa 
Cuadro 4. Pertinencia de los contenidos de las asignaturas del componente de investigación.

\begin{tabular}{|c|c|}
\hline Dimensión & Expresión discursiva de los estudiantes \\
\hline \multirow{8}{*}{$\begin{array}{l}\text { Pertinencia de los } \\
\text { contenidos de las } \\
\text { asignaturas del } \\
\text { componente de } \\
\text { investigación, }\end{array}$} & $\begin{array}{l}\text { "Yo tuve suerte que mi profa de Métodos I y Métodos II fue la misma y.... } \\
\text { bueno... ella si daba mucha práctica, para todo había un ejercicio. Que } \\
\text { si las fichas,...las citas... Luego de un planteamiento escribir, ...bueno...o } \\
\text { sea, redactar nosotros unos objetivos. Eso fue muy bueno,...ayuda,... } \\
\text { aprendes. Fue interesante junto con la práctica de diseño de instrumentos } \\
\text { de recolección de datos o sea un guión de entrevista, un cuestionario. Y } \\
\text { después... bueno análisis de eso que se anotaba alli. Fue muy } \\
\text { dinámico.(Agata) }\end{array}$ \\
\hline & $\begin{array}{l}\text { "Eso de que esas materias sean pura teoría no es bueno... Yo me aburría } \\
\text { de puras exposiciones de mis compañeros.... Hay no... no soportaba } \\
\text { esas clases..."(Zafiro). }\end{array}$ \\
\hline & $\begin{array}{l}\text { "No me gustó esas asignaturas...Pura teoría y en Investigación } \\
\text { Aplicada... vienen y te exigen que hagas un proyecto casi tu solo, porque } \\
\text { los tutores también te dan poco....Puro... está malo, corrija, pero no te } \\
\text { dicen cómo hacerlo bien...No sé...esa es mi experiencia actual...Shiiiiito } \\
\text { me guardan el secreto ... (risas) (Neptunita) }\end{array}$ \\
\hline & $\begin{array}{l}\text { “...es que podrían..Mmmmm, cómo decirlo... más unidas a las } \\
\text { asignaturas de la carrera. No sé cómo se haría, pero siempre pienso en } \\
\text { eso... es que...Mmmmm...investigación va por un lado y los contenidos } \\
\text { de Administración Comercial por otro...”" (Benotoita) }\end{array}$ \\
\hline & $\begin{array}{l}\text { “... Hay dos cosas...por un lado muchas cosas que te dan en Métodos } \\
\text { II... y por otra te piden que hagas un proyecto en Investigación } \\
\text { Aplicada,... pero, el profe de Investigación Aplicada no da ningún } \\
\text { contenido, ni refresca lo de Métodos I. Eso que la vimos seis o siete } \\
\text { semestres atrás y el contenido de Métodos II...es rudo!!!..., dificil. No es } \\
\text { fácil plantearse un problema o una interrogante de investigación... Yo no } \\
\text { sé cómo hacerlo y estoy ya en noveno semestre.”(Ambar). }\end{array}$ \\
\hline & $\begin{array}{l}\text { "Si eso de Investigación Aplicada es como... No sé... vaya Ud. solo y, } \\
\text { escriba y me trae. ¿Cuál es el contenido de esa materia?. Quién de uds lo } \\
\text { sabe?... vamos el genio de la lámpara que hable!!!...(Risas)”( Amatista) }\end{array}$ \\
\hline & $\begin{array}{l}\text { “...Investigación Aplicada es como la práctica de lo que vimos en la } \\
\text { teoría en Métodos II, más aquello de lo formal de Métodos I...pero lo } \\
\text { trabajas solo, porque el tutor...sobre eso...bien gracias!!..”(Peridoto) }\end{array}$ \\
\hline & $\begin{array}{l}\text { "En Investigación Aplicada, comienzan con: el capitulo I para tal } \\
\text { fecha..., el capitulo II para tal fecha ... y te quedas...Mmmm What? What? } \\
\text { ¿Cuándo dijo el profe qué era lo que contiene cada capítulo? De qué } \\
\text { habla? Sorry!!! Y salimos de clase locos... primer trauma para los que } \\
\text { siguen... (Risas)” (Perla). }\end{array}$ \\
\hline
\end{tabular}

Continúa 
Cuadro 4. Pertinencia de los contenidos de las asignaturas del componente de investigación.

\begin{tabular}{|c|c|}
\hline Dimensión & Expresión discursiva de los estudiantes \\
\hline \multirow{6}{*}{$\begin{array}{l}\text { Pertinencia de los } \\
\text { contenidos de las } \\
\text { asignaturas del } \\
\text { componente de } \\
\text { investigación, }\end{array}$} & $\begin{array}{l}\text { "Bueno... mi prof...de Investigación Aplicada nos decía el capítulo I } \\
\text { debe contener título, problema, interrogante y objetivos.... Eso lo dijo } \\
\text { ayer por cierto..."(Heliodor) }\end{array}$ \\
\hline & $\begin{array}{l}\text { “...Ves eso se contradice con lo que nos dice la profa de Métodos II, } \\
\text {...ella dice que el título es lo último que uno decide...”(Ambar). }\end{array}$ \\
\hline & $\begin{array}{l}\text { “...Mira eso es como una plantilla. Sigues en Investigación Aplicada lo } \\
\text { que te dieron en Método II. ...sigues cada uno de las etapas. Eso no tiene } \\
\text { nada,...el profe dice es así y así debe ser. Todo igual...”(Ópalo) }\end{array}$ \\
\hline & $\begin{array}{l}\text { "Todos los TEG es lo mismo, porque todos se rigen por un modelo. Yo } \\
\text { tengo tutor y él me dice, me dice... hazlo como te dijeron en Métodos II. } \\
\text { Todo es así... Sigue la norma...."(Onix) }\end{array}$ \\
\hline & $\begin{array}{l}\text { "Yo le pregunté a mi profe. de Investigación Aplicada ...¿itiene algún } \\
\text { trabajo suyo como modelo y me dijo: eso está en los libros...(Risas), O } \\
\text { sea no tiene ... Ves? Entonces ¿?...” (risa) (Nefrita) }\end{array}$ \\
\hline & $\begin{array}{l}\text { “...A mi me dieron investigación cualitativa y cuantitativa... A mí me } \\
\text { pareció bien la cualitativa..., pero cuando le dije al profe de } \\
\text { Investigación Aplicada que mi proyecto sería cualitativo ...no } \\
\text { hombre!!!...casi me tira al techo!!!!..que eso no!!...que pata tin...que } \\
\text { pata tan...que yo iba a ser contador público!!!, qué horror!!... Entonces, } \\
\text { para qué ve uno eso, si te obligan a hacerlo solo cuantitativo o como él } \\
\text { quiere...."(Onix) }\end{array}$ \\
\hline
\end{tabular}

Fuente. Grupo de discusión

A pesar que se tiene claro el propósito de estas asignaturas, en la malla curricular y en el ámbito formal, los estudiantes manifiestan cierto malestar en relación a dichas asignaturas. Esto queda expuesto en las opiniones discursivas de los grupos de discusión, que se recogieron en la tabla anterior. Un aspecto relevante que emergió fue lo relativo a la dinámica en el aula por parte del docente. Es decir, para los estudiantes, si bien consideraron que el contenido era muy importante y pertinente, este se diluye a consecuencia de la forma cómo se desarrollan las clases en dichas asignaturas. Es decir, de acuerdo a lo expresado por los estudiantes la mayoría de los docentes, de las asignaturas del componente de investigación, se limitan a repetir los contenidos de los libros, sin generar dinámicas en el aula que permitan aprender "haciendo". Según los estudiantes, los docentes no logran articular los contenidos teóricos que reciben, con el ejercicio de una práctica investigativa, lo cual en lugar de favorecer el acercamiento a la 
investigación científica, contrariamente genera en los estudiantes cierto desinterés y aburrimiento hacia estas asignaturas. Es importante acotar, que el interés por la investigación quedó manifiesto en aquellos estudiantes cuyos docentes les hacían participes en las actividades investigativas que desarrollaban y en cuyas clases se efectuaban ejercicios prácticos, para cumplir con cada tema del contenido. Esto reafirma que solo se puede enseñar a investigar investigando, tal como sostienen Martínez, 1989; Castro Silva, 1997; Sabino, 2000 y Arias, 2008. En efecto, se pudo constatar que aquellos docentes del componente de investigación que recurrían a realizar actividades prácticas, en el aula, eran los que se conocía que estaban desarrollando proyectos de investigación.

Esta realidad devela la importancia que tiene la formación como investigador del docente del componente de investigación en particular y de todo docente en general. Sólo contando con una buena experiencia investigativa los docentes pueden lograr acercar a sus estudiantes al mundo del conocimiento científico. Por ello, es necesario recurrir al lema formar-investigando e investigar-formando Yapu (2017), de modo que se pueda enriquecer su formación con la investigación (Páez, 2010) y estimular a los estudiantes al ejercicio de la investigación científica (Fernández, 2015).

En este sentido, vale acotar que en este proceso de acercamiento de los estudiantes universitarios, a su formación como investigadores, es importante que el docente no solo tenga dominio y experiencia investigativa, sino que, además, sea capaz de garantizar que ese proceso no se convierta en la simple reproducción de su esquema paradigmático, como única verdad incuestionable. Obligando al estudiante a mirar la realidad solo a través del lente por donde él la ve, coartando así cualquier otro procedimiento epistémico. Por el contrario, debe efectuarse desde un criterio amplio, critico, flexible e incluyente.

\section{Orientación académica para el diseño del proyecto de investigación}

Llama la atención que en los discursos emergió el problema de la necesidad de orientación al momento de incursionar en el proceso de investigación, esto queda evidenciado en los enunciados que se recogen en el Cuadro 5. 
Cuadro 5. Orientación académica para el diseño del proyecto de investigación

\begin{tabular}{|c|c|}
\hline Dimensión & Expresión discursiva de los estudiantes \\
\hline \multirow{7}{*}{$\begin{array}{l}\text { Orientación académica } \\
\text { para el diseño del } \\
\text { proyecto de } \\
\text { investigación }\end{array}$} & $\begin{array}{l}\text { “...Yo hice una monografia en Métodos I, aprendí a citar y buscar } \\
\text { información documental. La profe nos dio bases de datos electrónicas } \\
\text { donde hacer consulta. Eso lo valoro ahorita. Ahorita... } \\
\text { mira...yo...Busqué mis cuadernos de Métodos I... (risas).”(Oro) }\end{array}$ \\
\hline & $\begin{array}{l}\text { "Siempre tu oyes quejas de los compañeros sobre la falta de asesoría } \\
\text { para la parte metodológica...será porque muchos profes no investigan } \\
\text { tampoco??... Bueno mis profes de Métodos si me han ayudado mucho a } \\
\text { aclarar cosas de esas..." (Azabache) }\end{array}$ \\
\hline & $\begin{array}{l}\text { "Hay quienes dicen necesito un tutor metodológico y otro de contenido.., } \\
\text { pero mi profa de Métodos decía que eso no es correcto!! Porque el tutor } \\
\text { debe dominar todo!! Por eso también como ella decía: 'dominar el } \\
\text { abordaje } \quad \text { metodológico'(Risas) } \quad \text {...esas } \\
\text { palabras...Ojo"...(Risas)(Opalo) }\end{array}$ \\
\hline & $\begin{array}{l}\text { “...Pienso que necesito un tutor completo...o sea,... bueno que sepa de } \\
\text { métodos y de mi tema... sino es un rollo..."(Onix) }\end{array}$ \\
\hline & $\begin{array}{l}\text { "Mi experiencia en Métodos II fue malísima. Ese prof... No me dejó } \\
\text { nada..., hablaba y hablaba de cosas que aún no le encuentro sentido... } \\
\text { ¿Qué aprendí sobre el proceso de investigación en esa materia?...nada!!. } \\
\text { Eso me hizo tenerle idea a eso de hacer el TEG, para colmo... el tutor } \\
\text { tampoco me orienta en la parte metodológica. Él dice que losuyo es puro } \\
\text { contenido... ¿Entonces?? Acaso él no ha investigado??.."(Cuarzo) }\end{array}$ \\
\hline & $\begin{array}{l}\text { "Eso parece una lotería. Unos profes dan mucho ...importante... y eso... } \\
\text { de mucho valor para el proyecto y otros pasan por ahi como dicen... } \\
\text { esteeee.... un saludo a la bandera. Tienen que revisar... esas clases. ... tu } \\
\text { sabes que eso es muy importante para nosotros...porque una cosa es el } \\
\text { tutor que no quiere tocar lo de la metodologí....creo que le tienen } \\
\text { miedo(Risas)" (Lapizlasuli). }\end{array}$ \\
\hline & $\begin{array}{l}\text { "En Métodos } I, \text { yo aprendi bastante... yo no sabia lo que era buscar } \\
\text { información en revistas y libros y eso... documental pues!!... quiero decir } \\
\text { todo lo de la bibliografía. En Métodos II,... Mmmm, ya es lo de las etapas } \\
\text { de la investigación o lo del proyecto en sí... Yo sí recuerdo eso... pero otra } \\
\text { cosa es hacerlo... (Agata) }\end{array}$ \\
\hline
\end{tabular}

Continúa... 
Cuadro 5. Orientación académica para el diseño del proyecto de investigación

\begin{tabular}{|c|c|}
\hline Dimensión & Expresión discursiva de los estudiantes \\
\hline \multirow{4}{*}{$\begin{array}{l}\text { Orientación académica } \\
\text { para el diseño del } \\
\text { proyecto de } \\
\text { investigación }\end{array}$} & $\begin{array}{l}\text { "Yo olvidé todo lo de Métodos I cuando llegué a MétodosII. Es que..están } \\
\text { muy separados, además...! Y los tutores como que tampoco se acuerdan } \\
\text { i!(Risas)... porque tú les preguntas algo, por ejemplo de los objetivos, o } \\
\text { los indicadores y te dicen consulta con el prof.de Métodos... Porr } \\
\text { favorrr. Y el prof.de Métodos te manda con tu tutor porque no tiene } \\
\text { tiempo. Entonces???vamos a buscar "apoyo externo"... a pagar.. Qué } \\
\text { tal??"(Zafiro). }\end{array}$ \\
\hline & $\begin{array}{l}\text { "En Investigación Aplicada te exigen mucho, como si ya hubieras hecho } \\
\text { otros proyectos, iimaginate eso!!... Alli vas entregando capitulo por } \\
\text { capítulo. Pero se puede decir que vas solo. No hay clase de nada... No } \\
\text { hay aclaratoria de nada. Tienes que buscar "asesor" externo...Tu sabes } \\
\text { esa gente que te cobra ... Uffff!. No es justo. Esa asignatura debe tener un } \\
\text { contenido... cierto??? (Benotoita) }\end{array}$ \\
\hline & $\begin{array}{l}\text { Mi profe de Investigación Aplicada es lo máximo... porque él sabe } \\
\text { mucho. Es Contador y Administrador...Te orienta siempre, te corrige } \\
\text { tooodo...Si es exigente, pero es bueno...(Heliodor) }\end{array}$ \\
\hline & $\begin{array}{l}\text { Mi experiencia ha sido buena... Hasta ahora,... tanto mi tutor como los } \\
\text { profes de Métodos me han atendido... hasta han discutido entre ellos } \\
\text { sobre lo que opinan y me lo dicen para llegar a un acuerdo. Ha sido } \\
\text { chévere... voy avanzando bien... recibo orientación" (Agata) }\end{array}$ \\
\hline
\end{tabular}

Fuente: Grupos de discusión

De acuerdo a lo expuesto por los estudiantes, se pudo captar que, respecto a la orientación que reciben para el desarrollo del proyecto de investigación, afrontan situaciones que no contribuyen a generar interés por la investigación científica. Por el contrario entorpece el acercamiento al mundo de la ciencia.

Entre las debilidades fundamentales, que se develan del discurso, están por una parte la experiencia que vive al cursar las asignaturas del componente de investigación. Al respecto, salvo excepciones, revelan poca fortaleza en cuanto a los contenidos recibidos, producto de la forma como los profesores desarrollaban sus clases, así como el sentimiento de abandono luego que comienzan a perfilar su proyecto de investigación del TEG. Fundamentalmente, en la asignatura Investigación Aplicada, se revela que ni siquiera conocen el contenido programático de la asignatura, lo cual evidencia un fallo importante, pues independientemente que su propósito sea elaborar y desarrollar el Proyecto de TEG, es deseable que los estudiantes reciban algún tipo de refuerzo de lo recibido como formación en los contenidos de Métodos II, 
es decir, en la asignatura que le precede. Y por la otra, la cuestión del sentimiento de poco apoyo que reciben, desde el punto de vista metodológico, por parte de algunos de los docentes de esta asignatura, en el seguimiento de los proyectos de TEG.

Esto se revela cuando la mayoría de los estudiantes manifestaron que algunos tutores y docentes de Investigación aplicada no les acompañan, de forma rigurosa, en todo su transitar en la realización de su TEG. Además, se sienten abandonados en el limbo de la metodología, al no encontrar en sus docentes, ni en su tutor un espacio de encuentro para aclarar las dudas que normalmente se gestan durante el proceso de investigación. Ese acompañamiento es sumamente importante, sea como tutoría o asesoría (Gamero 2014, Castro-Gamero (2014), puesto que puede constituirse en la clave de la continuidad o no del proyecto de TEG, ya que

podría favorecer la ruptura con los miedos o temores que se arrastra de experiencias vividas por otros estudiantes (Herrera \& Sánchez, 2019).

Esta situación pudiera generar en los estudiantes desinterés en comprometerse en la actividad científica y confabular en la búsqueda de "auxilio" en entes externos que cobran cantidades considerables, por hacerles el trabajo investigativo. Es decir, este panorama alimenta la comercialización de los TEG, algo que ya es un secreto a voces.

\section{Divulgación de la actividad científica y sus productos}

La publicación de los productos de la investigación científica es fundamental para lograr divulgación de los saberes en las distintas disciplinas. De ahí que, los productos de la actividad investigativa tienen valor en la medida que éstos pueden ser compartidos en los escenarios de interés académico de cada disciplina. Esta temática emerge en los grupos de discusión como uno de los aspectos que los estudiantes revelan de gran interés en su acercamiento al mundo de la investigación, esto se evidencia en las expresiones discursivas contenidas en el Cuadro 6. 
Cuadro 6. Divulgación de la actividad científica y sus productos

\begin{tabular}{|c|c|}
\hline Dimensión & Expresión discursiva de los estudiantes \\
\hline \multirow{9}{*}{$\begin{array}{l}\text { Divulgación de la } \\
\text { actividad científica y } \\
\text { sus productos }\end{array}$} & $\begin{array}{l}\text { “... Es que un trabajo de grado sin norte no tiene sentido. Veo que te } \\
\text { enseñan sobre qué contiene y cómo manejar todas sus partes. ...pero, la } \\
\text { mayoría están engavetadas...no salen de la biblioteca..., quién se entera } \\
\text { que eso se hizo?". (Peridoto) }\end{array}$ \\
\hline & $\begin{array}{l}\text { "Yo te digo..., otra cosa sería... si fuera que vamos a escribir un artículo } \\
\text { con los resultados del proyecto. Quiero decir...como en Medicina que les } \\
\text { piden una versión tipo artículo cientifico. Yo no sé si se los publican... } \\
\text { pero se hace eso de intentar escribir un artículo científico... al menos. } \\
\text { "(Ambar) }\end{array}$ \\
\hline & $\begin{array}{l}\text { "Sí...yo preferiría hacer algo corto. Tanto trabajar para que nadie lo } \\
\text { lea...esteee... es como hacer algo para nada, ¿para ti?, ¿para tu } \\
\text { familia?. ¿Quién recibe el beneficio de tanto esfuerzo...? (Cuarzo) }\end{array}$ \\
\hline & $\begin{array}{l}\text { “.., pero no sería nada malo ver mi nombre en un artículo cientifico } \\
\text { (risas)...eso si es laaa nota”. (Topacio) }\end{array}$ \\
\hline & $\begin{array}{l}\text { "Conozco una profe que con sus tutoreados va a congresos, incluso } \\
\text { internacionales... eso si me entusiasma... Esa es la que incorpora a los } \\
\text { estudiantes en sus proyectos de investigación, Claro... asi sí." (Onix) }\end{array}$ \\
\hline & $\begin{array}{l}\text { "Mi profa de Métodos II nos mostró una publicación con sus } \\
\text { tutoreadas.... Me emocionó... ayyy sería bueniiiisimo"(Rutilio) }\end{array}$ \\
\hline & $\begin{array}{l}\text { "La institución debe impulsar la divulgación de nuestros trabajos de } \\
\text { grado, ... y los de los profes... los trabajos de ascenso de los profes, por } \\
\text { ejemplo ¿Dónde está eso?... porque en la Web de la UC esa información } \\
\text { no está actualizada. ..."(Nefrita) }\end{array}$ \\
\hline & $\begin{array}{l}\text { "Mira...Si los profes escriben poco, como te ponen a ti,...caramba!!..., } \\
\text { siendo un triste estudiante!!!,... a escribir no sé... sin la ayuda de ellos." } \\
\text { (Cuarzo) }\end{array}$ \\
\hline & $\begin{array}{l}\text { “...Creo que todos deberíamos llevar nuestros trabajos al Congreso de } \\
\text { nuestra facultad...para que se conozca.... ¿Verdad? sin miedo..." } \\
\text { (Azabache) }\end{array}$ \\
\hline
\end{tabular}

Fuente: Grupos de discusión

Resulta interesante develar que en el sentir de los estudiantes existe un significativo interés por la divulgación del producto que su quehacer científico, materializado en el TEG. Esta manifiesta preocupación se ha despertado en aquellos estudiantes que han podido tener contacto con docentes que estimulan a sus alumnos a participar en eventos científicos donde 
puedan llegar a exponer el producto de sus trabajos de investigación. Se desprende de este acercamiento que el docente tiene un importante rol cuando se tiene como propósito incentivar a los estudiantes universitarios a involucrarse con el quehacer científico durante su formación universitaria. Se desprende que los estudiantes que han tenido experiencias favorables con sus docentes del componente de investigación y sus tutores les genera mucha ilusión la posibilidad que su TEG pueda ser divulgado, bajo la modalidad de ponencia o artículo científico.

\section{Conclusiones}

Se logró captar que la resistencia de los estudiantes a la actividad investigativa, durante su formación universitaria, está anclada a la existencia de una brecha que se ha gestado entre la actividad investigativa que desarrollan los docentes en la institución y la actividad que los estudiantes realizan en sus TEG, lo cual entorpece la integración de los intereses investigativos de los docentes con los de los estudiantes, al no favorecer la participación e involucramiento mutuo en la actividad de investigación durante su proceso de formación universitaria.

Quedó manifiesto que los estudiantes no poseen información en relación a las actividades de investigación que desarrollan los docentes, en consecuencia se sienten al margen de dichas actividades. En ese sentido, perciben la actividad investigativa como un espacio restringido, donde solo acceden aquellos estudiantes que logran, de alguna manera, penetrar el espacio de los elegidos, porque pareciera existir la convicción que sólo pocos entran al mundo de la ciencia en la universidad.

Es decir, la institución no genera, a través de sus departamentos, centros, unidades de investigación y docentes, mecanismos de integración sistemática de los estudiantes a la actividad científica, haciéndoles partícipes de los proyectos que cada línea de investigación tiene en ejecución y las necesidades de incorporación de estudiantes en los mismos. Esta realidad limita la posibilidad que los estudiantes reconozcan como una cuestión normal, no excepcional, el hacer investigación durante su formación universitaria. Pues en la medida que esté insertado dentro de alguno de los grupos de investigación, lograría asumir la investigación como una actividad propia de su formación disciplinar, donde el TEG cobra valor. Esto redundaría en una vigorosa presencia de los estudiantes universitarios en eventos científicos (Congresos, Seminarios), así como en una activa participación en la publicación de artículos científicos. De modo que, la idea desvirtuada de la realización del TEG, como algo 
innecesario, como material para un repositorio donde nadie lo lee y por tanto sin mayor trascendencia, dejaría de ser un problema. Quedó revelada la alta valoración que los estudiantes le otorgan a la investigación científica, la cual es vista con admiración y como algo inaccesible pero deseado. Eso podría considerarse como un aspecto al cual se puede recurrir como fuente potenciadora del interés de los estudiantes hacia la actividad investigativa.

Al involucrar al estudiante en la actividad cientifica como eje transversal, no solo al momento de realizar su proyecto de investigación, éste podría desarrollar su TEG en el marco de una experiencia altamente generadora de satisfacciones, donde se ponga en valor su creatividad y potencial innovador, lo cual quebraría los miedos atávicos e ideas negativas preconcebidas respecto a lo que es investigar.

En este sentido, habría que hilar fino para lograr una urdimbre eficazmente resistente que logre mantener en estrecha alianza investigación y docencia, es decir producción intelectual y actividad académica. Donde docente-investigador y alumno se relacionen como una sola madeja para generar los productos que la ciencia demanda y que las instituciones universitarias están llamadas a liderar.

La resistencia de los estudiantes a la actividad investigativa, también se ha visto marcada por el hecho de sentir que su aproximación a la investigación se genera desde un cuerpo abstracto, de fundamentos teóricos, que son suministrados en las asignaturas del componente de investigación, como base fundamental para poder incursionar en el mundo de la investigación.

Es decir, se ha venido errando al asumir como modalidad la separación de los contenidos teóricos con el acto de investigar. Esta modalidad de enseñanza procura que los estudiantes, al momento de desarrollar su proyecto de investigación con fines de TEG, sientan profundos vacíos y la imperiosa necesidad del acompañamiento de un tutor o asesor que sea docente-investigador. Esto revela la importancia de esta figura durante el proceso. En consecuencia, la labor del tutor (docente-investigador) tiene una alta relevancia, para poder lograr que esa experiencia permita generar satisfacciones y potenciar la creatividad en los estudiantes, en un proceso que no coarte la iniciativa o deseos de búsqueda y abordaje diferente al esquema paradigmático donde el tutor se siente cómodo.

De hecho, a partir de una práctica amplia flexible, reflexiva y crítica cobraría sentido, en el imaginario de los estudiantes, la realización del TEG como acto investigativo y como 
factor que sirve de asiento, de base en la formación disciplinar. Es decir, podría ser visto como un mecanismo que le dará fortaleza y robustez al componente académico de los futuros Administradores Comerciales y Contadores Públicos, de modo que el TEG llegue a cumplir así su propósito dentro del pensum académico.

Ante esta realidad, surge la inquietud por indagar esta problemática desde el colectivo docente, de manera que se logre avanzar en las necesidades que afrontan los profesores, respecto a su formación como profesores investigadores en su área disciplinar. Esto podría incidir favorablemente en el proceso de enseñanza y aprendizaje de la investigación en la formación universitaria.

\section{Referencias}

Abreu, J. (2015). Síndrome Todo Menos Tesis (TMT). Daena: International Journal of Good Conscience. 10 (2) 246-259. ISSN 1870-557X.

Argüello, L. (2010). El concepto de la producción en la actividad académica del profesor universitario. Docencia Universitaria, 11, 89-105. Recuperado de http://revistas.uis.edu.co/index.php/revistadocencia/article/view/1913

Arias, F. (2008). El perfil del profesor de metodología de la investigación. Trabajo de Grado de maestría; no publicado, Universidad Central de Venezuela.

Barsky, O. (2017) Documentos sobre la educación superior. Wilhelm Von Humboldt Sobre la organización interna y externa de los establecimientos científicos superiores en Berlín (1810) Debate Universitario 6(11), 53-67

Bolívar, A. (2014). Actitudes de los estudiantes hacia la Asignatura Investigación Educativa. Aportes de la Teoría de la Valoración. Revista EDUCARE, Volumen 18, Número 2, ISSN: 2244-7296. (pp. 4-30)

Callejo, J. (2001). El grupo de discusión: introducción a una práctica de investigación. Barcelona, Ariel.

Castro-Gamero, (2014).La tutoría en pequeños grupos como recurso formativo para el aprendizaje del Derecho Romano. Revista de Docencia Universitaria REDU, 12 (3), $\mathrm{N}^{\circ}$ extraordinario, 269-292.

Castro-Silva, E. (1997). Bases para la enseñanza de la metodología de la investigación científica. Revista Pedagogía (12), 67-101.

Chacón, J. (2017). Impacto del componente de investigación en la orientación hacia la producción de conocimiento científico, a través del trabajo especial de grado, en los estudiantes de contaduría pública. Universidad de Carabobo. Valencia. Trabajo Especial de Grado. FACES, Universidad de Carabobo, Valencia.

Espig, H. y Silva, I. (2013). Evaluación de lo que opinan los estudiantes sobre el proceso de la realización del trabajo especial de grado. Salus 17(3), 51-61 
Fernández, A. (2015). Aprendizaje e Investigación un camino de mejoramiento y Fortalecimiento desde la construcción del Trabajo de Grado. Universidad de Carabobo, Valencia.

Flores \& Villegas (2008). Algunos elementos condicionantes del aprendizaje de la investigación en la educación superior: Caso UPEL Maracay. Investigación y Postgrado 23(1), 155-185.

Gamero, M. (2014). El proceso de acompañamiento en investigación desde la experiencia de tutores académicos. Trabajo de Grado de maestría; no publicado, Universidad de Carabobo, Bárbula.

González, M. (2014). La Investigación y su Aporte al Conocimiento en la Formación de Enfermería en la Facultad de Estudios Superiores Zaragoza de la Universidad Autónoma Nacional de México. Enfermería Universitaria 11(2):45-46

Herrera, M. \& Sánchez, L. (2019). El trabajo especial de grado como producto intelectual en la formación profesional. Una mirada a su significación desde el sentir de los estudiantes. Memorias. III Congreso Nacional de Investigación e Innovación de Ciencias Económicas y Sociales. FACES-UC.

Marrero, O y Pérez, M. (2014). Competencias investigativas en la educación superior. RES NON VERBA, Febrero.

Martínez, M. (1989). Comportamiento humano. México: Trillas

Martínez, O. J., Harrington M, M. S., \& Bolívar O, A. C. (2011). Concepciones asociadas al concepto de investigación en estudiantes de pregrado. Centro de Investigaciones Educativas (CIES), Venezuela.

Morales, O., Rincón, A \& Tona, J. (2005). Cómo enseñar a investigar en la universidad. Educere, 9(29), 217-225. Recuperado en 12 de marzo de 2020, de http://ve.scielo.org/scielo.php?script=sci_arttext\&pid=S131649102005000200010\&lng=es\&tlng=es.

Páez, J. (2010). La investigación universitaria y la formación del profesorado latinoamericano. Aposta. Revista de Ciencias Sociales, España. 4(7)1-38. [Fecha de consulta 17 de febrero de 2020].ISNN: Disponible en: https://www.redalyc.org/articulo.oa?id=4959/495950243004

Rodríguez-Méndez, M., González- Cantalapiedra, Z.\& González-Polo, M. (2016). Problemas frecuentes en la redacción de artículos científicos. EduSol, 6(57), 137-147.

Sabino, C. (2000). El proceso de investigación. Caracas: Panapo

Salinas, P. (1995). La relación entre el tutor y el tutorado. Editorial. MedULA, Revista de la Facultad de Medicina, Universidad de los Andes. Vol. 4 N 1-4. Mérida, Venezuela.

Sánchez, R. (2014). Enseñar a investigar Una didáctica nueva de la investigación en ciencias sociales y humanas. Universidad Nacional Autónoma de México. Instituto de Investigaciones sobre la Universidad y la Educación. ISBN: 978-607-02-5833-6, México.

Sivira, Y. (2014). Representaciones Sociales sobre la Investigación de los estudiantes del área de educación de la Universidad Nacional Experimental Francisco de Miranda, Universidad de Carabobo, Valencia. 
Valles, M. (2000). Técnicas cualitativas de investigación social. Madrid, Síntesis.

Yapu, M. (2017). Investigación y formación de investigadores. Algunas reflexiones epistemológicas a partir de una experiencia en Bolivia. Praxis Sociológica $\mathrm{n}^{\mathrm{o}} 22$. www.praxissociologica.ese-ISSN: 2174-4734 X. ISSN: 1575-08-17. Pp. 31-47. 